Serge Aberdam et alii, Voter, élire pendant la Révolution française 1789-1799. Guide pour la recherche

Paris, CTHS, Édition revue et augmentée, 2006, 573 p., ISBN

2-7355-0600-2, $32 €$.

\title{
Malcolm Crook
}

\section{(2) OpenEdition}

Journals

Édition électronique

URL : https://journals.openedition.org/ahrf/11425

DOI : $10.4000 /$ ahrf. 11425

ISSN : 1952-403X

Éditeur :

Armand Colin, Société des études robespierristes

Édition imprimée

Date de publication : 1 mars 2008

Pagination : 229-231

ISSN : 0003-4436

Référence électronique

Malcolm Crook, « Serge Aberdam et alii, Voter, élire pendant la Révolution française 1789-1799. Guide pour la recherche ", Annales historiques de la Révolution française [En ligne], 351 | janvier-mars 2008, mis en ligne le 29 décembre 2009, consulté le 05 août 2021. URL : http://journals.openedition.org/ahrf/11425 ; DOI : https://doi.org/10.4000/ahrf.11425 
citoyens-combattants : le " rétablissement du modèle capacitaire de la citoyenneté " (p. 93).

Les autres communications, d'intérêt parfois plus inégal, apportent néanmoins quelques éclairages sur la construction de l'" homme politique " en Révolution. Larticle de Christine Le Bozec, dont les travaux sur Boissy d'Anglas avaient apporté de nombreuses réponses à l'anomalie historiographique que constituait la carrière des libćraux il y a un peu plus d'une dizaine d'années, est un court rappel de la conception libérale de l'" homme politique " au tournant du XIX" siècle, à la fois attachée aux libertés, mais acceptant le recours temporaire à un pouvoir fort lorsqu'il s'agit de garantir la stabilité de l'État. La communication d'Olivier Blanc est unc reprise de ses publications antérieures sur Olympe de Gouges (Marie-Olympe de Gouges, une humaniste à la fin du XVII siècle, Paris, Éditions René Viénet, 2003). Même si les remarques sur les différentes formes d'engagements de cette femme en politique sont loin d'être inutiles, on trouvera surtout ici une vision interne qui tend parfois un peu trop à la réhabilitation. On regrettera enfin que la contribution de Jean-Philippe Chimot, a priori incontournable sur un tel sujet (" David a-t-il contribué à constitucr la figure de l'« homme politique »?»), ne s'insère pourtant que partiellement dans la problématique d'ensemble, qui appelait peut-être davantagc une réflexion d'histoire intellectuelle et sociale sur les réseaux politiques et artistiques du député-peintre ou peintre-député, et/ou bien des analyses iconographiques vraiment détaillées d'un panel d'œuvres. La fin de l'article, consacrée au rôle de David dans la construction politique de Napoléon, comble en partie cette déception.

La nature même de ce genre de publication, qui vise évidemment avant tout à lancer des pistes et confronter les points de vue, tempère d'emblée ces quelques critiques. Lensemble offre une proposition stimulante. Celle-ci présente à la fois le mérite de rendre compte de certains travaux novateurs dans le champ de l'histoire politique et d'élargir la focale en réponse à une question contemporaine sur le rôle de la durée des carrières politiques dans la crise démocratique.

Guillaume MAZEAU

Serge ABERDAM et alii, Voter, élire pendant la Révolution française 17891799. Guide pour la recherche, Paris, CTHS, Édition revue et augmentće, 2006, 573 p., ISBN 2-7355-0600-2, $32 €$.

On doit saluer la deuxième édition de ce guide précicux, qui n'était plus disponible. Le relire nous rappelle ses richesses, avant tout ses séries de textes concernant les élections. Une collection complète des lois et décrets y est comprise, de la constitution des municipalités en 1789 jusqu'à l'ouverture des registres pour l'émission des votes sur la Constitution de l'an VIII, qui limite sévèrement le rôle du processus électoral. Accompagnés d'une trentaine de documents - procèsverbaux, protestations, candidatures - ces textes originaux composent l'essentiel de l'ouvrage (autour de 400 pages). La notice sur l'état des sources d'archives est aussi très utile, tout comme l'excellente mise au point de Bernard Gainot sur l'historiographie et l'histoire de la pratique électorale, qui pose les questions tout autant 
qu'elle souligne les acquis.

En l'espace de vingt ans, l'étude des élections est passée de parent pauvre de la recherche sur la Révolution au statut de riche héritier. On possédait alors des travaux sur les parlementaires, voire sur le profil socio-professionnel des responsables locaux ; bref, on était bien renseigné au sujet des élus. En revanche, les études de l'esprit public remontant au début du $\mathrm{XX}^{\mathrm{c}}$ siècle mises à part, il nous manquait des recherches sur la manière de voter pendant la décennie révolutionnaire. C'est le mérite de J.-R. Suratteau d'avoir attiré l'attention sur cette lacune dans un article paru en 1968. Mais plutôt que la sociologie de ces élections - grande préoccupation des politologues qui traitent le phénomène à partir de la Troisième République l'intérêt est bientôt porté sur l'acte électoral lui-même : comment on vote, en assembléc, sous la Révolution. Ce sont là des problèmes posés dans une optique de culture politique. Une vingtaine d'années plus tard, les élections de la période révolutionnaire ne sont plus négligées.

Et pourtant, on aurait dû mieux saisir l'opportunité d'une deuxième édition de ce guide électoral, finalement peu revu et augmenté. On n'y a ajouté qu'un document sur les pratiques et comportements : un procès-verbal d'élection d'une supérieure et d'une économe dans un couvent de l'Oise en février 1791, qui présente un rare exemple d'un vote de femmes (aussi évident dans le vote sur la Constitution de 1793, ou le partage des biens communaux). Parmi les textes officiels on retrouve ćgalement une seule addition : le décret du 31 décembre 1790 sur les classes de gens de mer, qui sont appelés à élire les syndics chargés de s'occuper de leur service public. Lexemple, cependant, souligne la large application du principe électoral sous la Révolution, qui englobe non seulement le personnel municipal ou départemental, mais aussi les positions ecclésiastiques, militaires, judiciaires et professionnelles, sans parler des "plébiscites". Une absence, peut-être, est à signaler : celle des "électeurs " du second degré, qui jouaient un rôle très important aux assemblées départementales tout le long des années 1790.

Il est dommage aussi qu'on n'ait pas plus augmenté la bibliographie des sources secondaires. Certes, les grands titres sont présents, y compris les travaux récents des deux Serge, Aberdam et Bianchi, ou de Jeff Horn sur l'Aube et de Philippe Tanchoux sur les procédures électorales. Cette liste impressionnante comprend aussi des travaux universitaires inćdits, mais non moins essentiels. En revanche, elle omet nombre de communications en français ou en anglais, souvent publiées dans des actes de colloques difficiles à trouver. Une même remarque peut être faite à propos d'études parues dans les revues, outre-Manche et outre-Atlantique, aussi bien qu'en France. Il manque également une indication des cartes électorales.

Le hibou de Minerve vole véritablement au crépuscule et on peut se demander si cette deuxième édition du guide paraît au moment où le grand jour des travaux électoraux est passé. Serge Aberdam, conscient de l'importance du chantier dès 1991, remarque dans son avant-propos que l'atelier qu'il a fondé pour l'étude des élections « ayant rempli son office, celui-ci décide en 2003 de tirer sa révérence ". Célébration donc, plutôt qu'invitation à la recherche ? Peut-être pas. Un numéro récent de Provence historique (228) comprend deux articles consacrés aux élections et l'un des deux auteurs, Cyril Belmonte, vient de soutenir une thèse sur les classes dirigeantes de l'arrière-pays marseillais dans laquelle il s'occupe beaucoup des 
votants. De plus, on attend toujours le grand livre de Melvin Edelstein, qui a voyagé dans toute la France en quête des données électorales.

Ce qui est certain, c'est que ce guide représente le résultat des grandes recherches, menées pour la plupart au cours des années 1990 et dont l'impact se mesure dans la référence obligatoire aux élections qu'on retrouve actuellement dans tous les manuels sur la Révolution. On a souvent mis en évidence le niveau d'abstention et parfois les violences qui accompagnent ces concours électoraux, mais comment ne pas souligner que cet apprentissage électoral au suffrage élargi, le premier dans le monde, a concerné des millions de Français ? Grâce au mécanisme d'assemblée, avec ses votes multiples, les citoyens d'alors ont participé à la vie publique d'une manière beaucoup plus profonde que les citoyens d'aujourd'hui. L'activité traitée dans ce guide indispensable marque ainsi tout à la fois l'origine et un âge d'or de la pratique des élections.

Malcolm CrooK

Thérèse Rouchetre (présenté par), Femmes oubliées de la guerre de Vendée, La Roche-sur-Yon, Centre vendéen de recherches historiques, 2005, 363 p., ISBN 2-911253-26-4, $24 €$.

Thérèse Rouchette présente, dans cet ouvrage, les mémoires de huit Vendéennes : Renée Bordereau « la combattante ", Sophie de Sapinaud " la belle Vendéenne ", Marie Lourdais "la providentielle », Françoise Després « militante et mythomane ", Pauline de Cambourg « femme d'émigré », Pauline Gontard des Chevalleries « la fugitive ", Julienne de Boishéraud « la rescapéc des noyades", Marie Trichet "l'enfant témoin de l'horreur".

Le livre est publié dans la collection mémoire de Vendée par le Centre vendéen de recherches historiques dont le comité scientifique a comme président fondateur François Furet et Pierre Chaunu parmi les présidents en exercice. Son conseil scientifique comporte, par ailleurs, de nombreux historiens spécialistes de la Vendéc tcls Roger Dupuy et Claude Petitfrère.

Lobjectif de cet ouvrage est de montrer la grandeur d'âme que les femmes de Vendée ont montré dans l'adversité en publiant les récits par lesquels elles auraient, plus tard, voulu témoigner.

Les sources présentées et annotées par Thérèse Rouchette ont des statuts différents, l'attribution de ces mémoires aux femmes qui sont supposées les avoir écrits est parfois délicate. Il s'agit de deux nobles, deux bourgeoises et quatre femmes du peuple. Ces dernières à part Françoise Desprée ne savaient pas écrire, des doutes existent sur l'existence de l'une d'entre elles. Les deux nobles écrivent tardivement et sont très âgécs. Thérc̀se Rouchette ćvoque, dans l'introduction, les multiples interrogations posées par les documents, et y revient à l'occasion de l'épilogue. Elle montre que ces brochures de propagande ont été diffusées sous la Restauration, mais la critique des sources, pourtant fondamentale pour ce type de documents, est menée un peu rapidement. Thérèse Rouchette ne s'appuie que très rarement sur les travaux d'historiens qui auraient pu enrichir ses analyses. Louvrage souffre évidemment de 